\title{
The downstream modulator of interferon- $\gamma$, STAT1 is not genetically associated to the Dutch coeliac disease population
}

\author{
Begoña Diosdado ${ }^{1,4}$, Alienke J Monsuur ${ }^{1,4}$, María Luisa Mearin ${ }^{2}$, Chris Mulder ${ }^{3}$ and \\ Cisca Wijmenga*,1
}

${ }^{1}$ Complex Genetics Section, Department of Biomedical Genetics, University Medical Centre Utrecht, Utrecht, The Netherlands; ${ }^{2}$ Pediatric Gastroenterology, Leiden University Medical Center, Leiden, The Netherlands; ${ }^{3}$ Department of Gastroenterology, VU University Medical Centre, Amsterdam, The Netherlands

Coeliac disease (CD) is a complex genetic disorder. Its etiology is owing to multiple genes and environmental factors, such as gluten. The first event in the pathogenesis of CD after the ingestion of gluten is the activation of a Th1 immune response that leads to villous atrophy. Although this immune response seems crucial to the disease's development, only the HLA-DQ2/DQ8 genes have been identified as causative immune genes related to CD. Recently, the activation of the transcription factor STAT1 and changes in its expression levels have confirmed the participation of the Janus kinase-signal transducer and activator of transcription pathway in CD. Furthermore, as the STAT-1 gene is a positional candidate located in the CELIAC3 locus on chromosome 2, we speculate that alterations in this gene could be primarily responsible for the aberrant immune response that characterizes $C D$. Based on this functional and genetic evidence, we investigated the primary contribution of STAT-1 to CD. We performed a comprehensive genetic association study using five tag SNPs fully covering the STAT-1 gene in a Dutch cohort of 355 independent $C D$ cases and 360 healthy controls. Neither the alleles, nor the genotypes in the case-control genetic association studies, nor the haplotype analysis showed any association to the STAT-1 gene in the Dutch CD population. Our results do not point to a primary involvement of the STAT-1 gene in the Dutch CD population.

European Journal of Human Genetics (2006) 14, 1120-1124. doi:10.1038/sj.ejhg.5201667; published online 14 June 2006

Keywords: coeliac disease; Th1 immune response; JAK-STAT pathway

Introduction

Coeliac disease (CD) is a complex genetic disorder in which the main causative environmental factor is the dietary protein gluten. This is present in common Western foods, such as bread, pasta and many processed products. When

\footnotetext{
*Correspondence: Dr C Wijmenga, Complex Genetics Section, Department of Biomedical Genetics, Stratenum 2.117, University Medical Centre Utrecht, PO Box 85060, 3508 AB Utrecht, The Netherlands.

Tel: + 3130253 8427; Fax: + 3130253 8479;

E-mail: t.n.wijmenga@med.uu.nl

${ }^{4}$ These two authors contributed equally to this work.

Received 9 November 2005; revised 11 April 2006; accepted 25 April 2006; published online 14 June 2006
}

gluten is ingested by coeliac patients, it provokes an inflammatory reaction in the small intestine that leads to crypt hyperplasia and villous atrophy. ${ }^{1}$ Although the exact nature of this immune response remains unknown, there is much evidence to suggest that gluten promotes a Th1 immune response mostly mediated by high levels of interferon-gamma (IFN- $\gamma)^{2}$ and $\alpha \beta$ CD $4+\mathrm{T}$ cells that recognize gluten peptides through HLA-DQ2/DQ8 molecules. $^{3}$ This observation is further supported by genetic data showing a primary association between the genes encoding the HLA-DQ2/DQ8 molecules on chromosome 6 and all the CD populations tested to date. ${ }^{4,5}$ Despite the fact that these results indicate an essential role for HLA in 
the etiopathogenesis of CD, the HLA-DQ2/DQ8 genes only explain approximately $40 \%$ of the familial clustering to $\mathrm{CD},{ }^{5,6}$ implying that other non-HLA genes must also be associated with CD. As the Th1 immune response is such a crucial factor in the pathogenesis of CD, the search for other primary genes should focus on genes linked to the Th1 immune response.

The Janus kinase-signal transducer and activator of transcription (JAK-STAT) pathway plays an important role in regulating the immune response. This pathway mediates the early Th1 proinflammatory IFN- $\gamma$-directed response by activating gene transcription through different STAT proteins (Figure 1a). The activation of an aberrant Th1 immune reaction initiated by the presence of gluten is a crucial event in the pathogenesis of CD. ${ }^{7}$ Some studies have shown the importance of the JAK-STAT pathway in the pathogenesis of CD. ${ }^{8-10}$ One interesting activator of the JAK-STAT pathway in CD might be IFN- $\gamma$, which is also highly expressed in the intestine of coeliac individuals. ${ }^{2}$ The exact role of IFN- $\gamma$ in the pathogenesis of $\mathrm{CD}$ has not been elucidated, but it might contribute to the maintenance of the chronic inflammatory response through the JAK-STAT pathway by regulating the expression and the activation of STAT1.

Although IFNG might play an important role in CD pathogenesis, ${ }^{2}$ genetic association studies have failed to show that this gene makes a primary contribution to the disease. ${ }^{2,11}$ Hence, it seems attractive to test the STAT1 gene, as it is one of IFN- $\gamma$ first downstream targets. The STAT1 gene is both a positional and functional candidate gene. The STAT1 gene is located in the CELIAC3 locus on chromosome $2 \mathrm{q} 23-32,{ }^{12}$ which also encompasses the
ICOS-CTLA4-CD28 gene cluster. Several studies have investigated the contribution of the CTLA4 gene in CD as this locus has been repeatedly shown to be associated with autoimmune disorders. ${ }^{13}$ However, the genome-wide linkage study performed in the Dutch population did not show significant linkage to this region. ${ }^{5}$ Initial genetic association studies on the CTLA4 gene in the Dutch population showed borderline significance for the CT60-G allele $(P=0.048)$, but this did not remain significant after correcting for multiple testing ${ }^{14}$ and could also not be replicated in an expanded cohort. ${ }^{15}$ Moreover, the weak association reported by some groups is insufficient to explain the linkage peak. It is likely that there is another gene from this region associated with CD susceptibility. Furthermore, linkage studies performed in this region have shown that the markers with the highest significant values are located outside the ICOS/CTLA4/CD28 gene boundaries and toward the centromeric side of the ICOS/CTLA4/CD28 genes, where the STAT1 gene is located ${ }^{4,12,16}$ (Figure 2). The STAT1 gene is located $12.9 \mathrm{Mb}$ centromeric to the CTLA4 gene, $3.7 \mathrm{Mb}$ from D2S117, which was identified in a metaanalysis performed in European populations $(P=0.023),{ }^{4}$ 2.2 Mb from D2S1776, which showed an lod score of 2.3 in a genome-wide linkage study carried out in the Finish population $^{12}$ and $10.9 \mathrm{Mb}$ from D2S2214 found in a metaanalysis that included five North European population $(P=0.0001)^{16}$ (Figure 2).

From a functional point of view, the STAT1 protein has been shown to be activated in the mononuclear cells of the lamina propria and epithelial cells of CD biopsies. ${ }^{8}$ These findings are further substantiated by mRNA expression

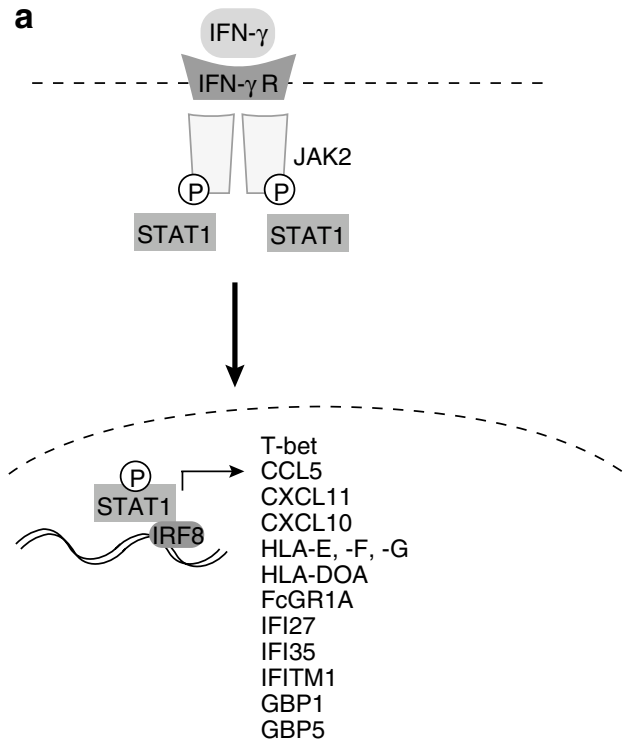

\begin{tabular}{llc}
$\begin{array}{ll}\text { b } \\
\text { Gene symbol }\end{array}$ & \multicolumn{1}{c}{ Gene name } & Fold changes \\
\hline IFNG & Interferon gamma & 0.1 \\
STAT1 & Signal transducer and activator of transcription 1 & 0.51 \\
JAK2 & Janus kinase 2 & 0.75 \\
IRF8 & Interferon regulatory factor 8 & 0.8 \\
T-bet & T-box 21 & 0.65 \\
CCL5 & Chemokine (C-C motif) ligand 5 & 0.63 \\
CXCL10 & Chemokine (C-X-C motif) ligand 10 & 0.49 \\
CXCL11 & Chemokine (C-X-C motif) ligand 11 & 0.05 \\
HLA-E & HLA-E histocompatibility antigen, class I, E & 0.65 \\
HLA-F & HLA-F histocompatibility antigen, class I, F & 0.58 \\
HLA-G & HLA-G histocompatibility antigen, class I, G & 0.66 \\
HLA-DOA & Major histocompatibility complex, class II, DO alpha & 0.76 \\
FCGR1A & Fc fragment of IgG, high affinity la, receptor (CD64) & 0.79 \\
IFI27 & Interferon, alpha-inducible protein 27 & 0.34 \\
IFI35 & Interferon, alpha-inducible protein 35 & 0.79 \\
IFITM1 & Interferon induced transmembrane protein 1 & 0.76 \\
GBP1 & Guanylate binding protein 1, interferon-inducible & 0.52 \\
GBP5 & guanylate binding protein 5, interferon-inducible & 0.72
\end{tabular}

Figure 1 Main components of the JAK-STAT pathway and differentially expressed genes in our expression studies. (a) The JAK-STAT pathway mediates the early Th1 proinflammatory IFN- $\gamma$-directed response by activating gene transcription through the STAT1 protein. (b) mRNA expression levels of the STAT1 gene, IFNG, as well as multiple downstream targets of the JAK-STAT1 cascade on biopsies of CD patients. 


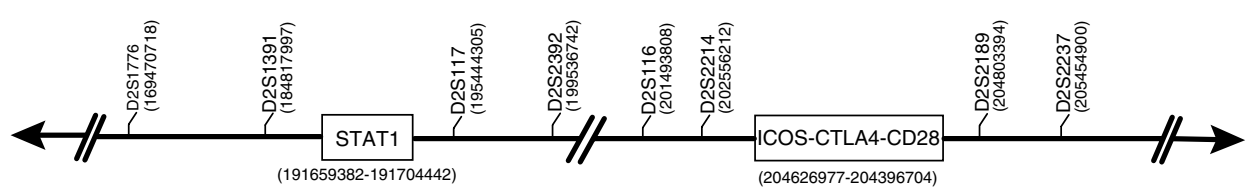

Figure 2 Overview of the markers and their location in the CELIAC3 region. These have been obtained from three reported studies in CD. ${ }^{4,12,16}$ The position of the markers and genes is given in bps according to Ensembl v37 Feb 2006. The figure is not drawn to scale.

studies on biopsies of CD patients, with upregulation of the STAT1 gene, IFNG, as well as multiple downstream targets of this signal-transduction cascade (Figure 1b) (Diosdado et al in preparation). These observations suggest that STAT1 might be critical in controlling the immunological activity in $\mathrm{CD}$ and, hence, any genetic change that alters its transcriptional activity could explain the detrimental immune response seen in CD. Based on this evidence, we carried out a comprehensive approach using tag SNPs to investigate the genetic contribution of the non-HLA gene STAT1 in the Dutch CD population in a case-control cohort.

\section{Subjects and methods}

\section{DNA samples for genetic association studies}

For the genetic association study, we collected a group of 355 independent CD cases and 360 independent blood bank controls, all of Dutch Caucasian origin. All CD patients were diagnosed according to the ESPGHAN criteria $^{17}$ and were selected for showing a Marsh III lesion at initial diagnosis. Ninety-three percent of the patients were HLA-DQ2 positive. We collected blood samples and isolated DNA according to standard laboratory procedures. $^{5}$

Informed consent was obtained from all individuals and the study was approved by the Medical Ethics Committee of the University Medical Centre, Utrecht, The Netherlands.

\section{Power calculations}

We have more than $80 \%$ power to detect a disease variant with minor allele frequencies ranging from 0.1 to 0.3 , assuming a relative risk of 1.7 , a dominant inheritance, a disease prevalence of the 0.1, a $D^{\prime}$ of 1 and an equal frequency of the tested SNP and the high-risk variant. In case of a different frequency of the high-risk allele compared to the tested SNP or an imperfect tagging, the power drops. In case of a codominant effect, the power increases.

\section{SNP selection and data analysis}

To select the tag SNPs, we downloaded the genotype information on the SNPs for the STAT1 gene from the HAPMAP database release 18 phase I (http://www.hapmap. org/) (typed in the CEPH population of Utah residents with ancestors from Northern and Western Europe). We uploaded this to the Tagger program (http://www.broad. mit.edu/mpg/tagger/). This software returned six nonredundant tag SNPs with a minor allele frequency (MAF) $\geqslant 10 \%$ and an $r^{2} \geqslant 0.8$ that covered the STAT1 gene. For these SNPs, two assay-on-demand probes: rs3771300 (C_22274194_10) and rs1914408 (C_1737196_10); and four assay-by-design probes: rs7562024 (C_1737208_10), rs1547550 (C_1737199_10), rs13010343 (C_1737197_10) and rs2030171 (C_1737225_20) were obtained. All the tested SNPs were analyzed on an ABI Prism 7900 HT system. All reagents and equipment were purchased from Applied Biosystems (Foster, CA, USA) and used according to their protocols.

Hardy-Weinberg equilibrium was evaluated separately in the case and control groups, for all tested SNPs (data not shown). Differences in allele frequencies and genotypes were compared between cases and controls using a $\chi^{2}$ test.

\section{Results}

To study the STAT1 gene as a causative factor in CD, we selected six tag SNPs for further genetic association studies (Table 1). Although the SNP rs 1547550 could not be tested owing to manufacturing failures, the other five tag SNPs captured $90 \%$ of the untyped SNPs present in the gene with an $r^{2}>0.8$ and MAF $>10 \%$. All SNPs were in HWE (data not shown). The association studies showed no statistical difference between the cases and controls for either allele (Table 1a) or genotype frequencies (Table 1b). Haplotype analysis did not improve the results (data not shown). We therefore concluded that the STAT1 gene is not associated to $\mathrm{CD}$ in the Dutch population.

\section{Discussion}

The CELIAC3 region has been found to be significantly linked to CD in the Scandinavian population and was also reported in a meta-analysis carried out by a European consortium on CD. ${ }^{4,12}$ This locus contains a number of interesting candidate genes for $\mathrm{CD}$, but so far attention has mainly been focused on the CTLA4 gene, as it has shown association to other autoimmune disorders. ${ }^{13}$ Nevertheless, genetic studies in $\mathrm{CD}$ have been rather inconclusive and do not clearly point to a genetic association with 
Table 1 -values obtained from the genetic association study in the STAT1 gene using tag SNPs on 355 CD patients and 360 controls. (a) Tag SNP information and association studies on allele frequencies. (b) Association studies on genotype frequencies

\begin{tabular}{|c|c|c|c|c|c|c|c|}
\hline SNP rs number & Bp position & SNP type & $M A F^{\mathrm{a}}$ & Cases (\%) & Controls (\%) & $\chi^{2}$ & $P$-value \\
\hline \multicolumn{8}{|l|}{ (a) } \\
\hline rs3771300 & 191661102 & $\mathrm{~T} / \mathrm{G}$ & G & 48.0 & 48.5 & 1.78 & 0.1821 \\
\hline rs1914408 & 191665482 & $\mathrm{C} / \mathrm{T}$ & $\mathrm{T}$ & 25.5 & 24.9 & 0.078 & 0.7807 \\
\hline rs13010343 & 191668951 & $\mathrm{~A} / \mathrm{G}$ & A & 15.8 & 12.4 & 3.36 & 0.0668 \\
\hline rs7562024 & 191681027 & $\mathrm{C} / \mathrm{T}$ & $\mathrm{T}$ & 35.9 & 37.1 & 0.226 & 0.6344 \\
\hline rs 2030171 & 191694669 & $\mathrm{~A} / \mathrm{G}$ & A & 32.9 & 31.8 & 0.206 & 0.6502 \\
\hline SNP rs number & Genotype & Cases (\%) & Controls (\%) & $\chi^{2^{\mathrm{b}}}$ & $P$-value ${ }^{\mathrm{b}}$ & & \\
\hline \multicolumn{8}{|l|}{ (b) } \\
\hline \multirow[t]{4}{*}{ rs37771300 } & GG & $89(25.6)$ & $96(26.8)$ & & & & \\
\hline & GT & 155 (44.7) & $177(49.4)$ & & & & \\
\hline & $\mathrm{TT}$ & $103(29.7)$ & 85 (23.7) & & & & \\
\hline & & & & 1.68 & 0.19 & & \\
\hline \multirow{3}{*}{ rs1914408 } & $\mathrm{CC}$ & $194(55.9)$ & $200(56.8)$ & & & & \\
\hline & $\begin{array}{l}\mathrm{CT} \\
T T\end{array}$ & $\begin{array}{c}129(37.2) \\
24(6.9)\end{array}$ & 129 (36.6) & & & & \\
\hline & & (ס.2) & $20(0.0)$ & 0.00 & 0.96 & & \\
\hline \multirow{4}{*}{ rs13010343 } & AA & $250(71.8)$ & $271(77.2)$ & & & & \\
\hline & $A G$ & $86(24.7)$ & $73(20.8)$ & & & & \\
\hline & GG & $12(3.4)$ & $7(2.0)$ & & & & \\
\hline & & & & 1.64 & 0.20 & & \\
\hline \multirow[t]{4}{*}{ rs7562024 } & $\mathrm{CC}$ & 145 (41.8) & $142(40.7)$ & & & & \\
\hline & CT & 155 (44.7) & 155 (44.4) & & & & \\
\hline & TT & 47 (13.5) & 52 (14.9) & & & & \\
\hline & & & & 0.03 & 0.87 & & \\
\hline \multirow{4}{*}{ rs2030171 } & AA & $35(10.2)$ & $38(10.9)$ & & & & \\
\hline & $A G$ & $155(45.3)$ & 145 (41.7) & & & & \\
\hline & GG & $152(44.4)$ & $165(47.4)$ & & & & \\
\hline & & & & 0.24 & 0.63 & & \\
\hline
\end{tabular}

${ }^{a}$ Minor allele frequency.

${ }^{\mathrm{b}}$ Overall $\chi^{2} P$-value of all three genotypes.

CTLA4. ${ }^{12,14-16,18-29}$ Therefore, it is tempting to speculate that another gene in this region may be the causative one in $\mathrm{CD}$ pathogenesis. In this study, we selected as a candidate the STAT1 gene. Its protein is one of the main downstream modulators of IFN- $\gamma$ signaling through the JAK-STAT pathway. The STAT1 gene is located in the CELIAC3 region on chromosome $2 \mathrm{q} 23-32$, and its participation in the pathogenesis of $C D$ is suggested by persistent activation at the lamina propria, and the upregulation of IFNG, several components of the JAK-STAT pathway and its downstream targets. ${ }^{8-10}$

We therefore conducted genetic association studies in a Dutch case-control cohort to determine the contribution of the STAT1 gene in the etiology of CD. Our association studies did not reach statistical significance for any of the tested tag SNPs. Although we cannot exclude a minor effect of this gene in the Dutch population, our results gave no evidence for STAT1 being a strong causative factor in the Dutch CD population.

Despite the results from the genetic studies on IFNG and STAT1, the importance of the JAK-STAT1 pathway in the immune response to gluten is still remarkable. However, at this point, we cannot exclude that upregulation of the downstream STAT-1 targets is secondary to the high IFN- $\gamma$ production in the lamina propria of CD patients. Unfortunately, there have been no functional experiments to determine the exact nature of the stimuli that initiate the JAK-STAT1 pathway in CD. It would be logical to assume that the major stimulator of this pathway is IFNG, as large amounts of it are seen in the biopsies of CD patients, but other cytokines such as IL-6 can also activate this pathway. $^{30}$ Additional studies on the JAK-STAT1 pathway, revealing the range of genes regulated by this transcription factor under the presence of gluten, are important, and should reveal new aspects of the immune response that occurs in CD. We still need to determine whether other STAT (STAT1-6) and JAK proteins are activated in the coeliac intestine, and especially, which molecules of the regulatory mechanisms of this pathway (the suppressor of cytokine signaling (SCOCS) family, the protein inhibitor of activated STAT (PIAS) proteins and protein tyrosine phosphatases (PTPs)) participate in the pathogenesis of $\mathrm{CD}$. Interestingly, some of the molecules of the regulatory mechanisms have already been related to immune diseases, like the regulatory molecule protein, tyrosine phosphatase receptor type $\mathrm{C}(P T P R C)$ that has been associated to 
multiple sclerosis. ${ }^{31}$ Identifying other members of the JAK-STAT pathway participating in CD, and the molecules that maintain its balance, might therefore reveal potential candidate genes for further genetic association studies in CD.

In conclusion, although functional studies imply that STAT1 plays an important role in regulating the extensive activation of the adaptive immune response in the lamina propria of $C D$, genetic association studies do not show any significant association of this gene to the Dutch CD population.

\section{Acknowledgements}

We thank Jackie Senior for editing the manuscript and the lab members of the CD group for fruitful discussions. We thank the Netherlands Organization for Medical and Health Research (ZonMW, Grants 902-22-094 and 912-02-028), and the Coeliac Disease Consortium, an innovative cluster approved by the Netherlands Genomics Initiative and partially funded by a Dutch government grant (BSIK03009), for financial support.

\section{References}

1 Marsh MN: Gluten, major histocompatibility complex, and the small intestine. A molecular and immunobiologic approach to the spectrum of gluten sensitivity ('celiac sprue'). Gastroenterology 1992; 102: 330-354.

2 Wapenaar MC, van Belzen MJ, Fransen JH et al: The interferon gamma gene in celiac disease: augmented expression correlates with tissue damage but no evidence for genetic susceptibility. J Autoimmun 2004; 23: 183-190.

3 Sollid LM: Coeliac disease: dissecting a complex inflammatory disorder. Nat Rev Immunol 2002; 2: 647-655.

4 Babron MC, Nilsson S, Adamovic S et al: Meta and pooled analysis of European coeliac disease data. Eur J Hum Genet 2003; 11: $828-834$

5 Van Belzen MJ, Meijer JW, Sandkuijl LA et al: A major non-HLA locus in celiac disease maps to chromosome 19. Gastroenterology 2003; 125: 1032-1041.

6 Bevan S, Popat S, Braegger CP et al: Contribution of the MHC region to the familial risk of coeliac disease. J Med Genet 1999; 36: 687-690.

7 Sollid LM: Molecular basis of celiac disease. Annu Rev Immunol 2000; 18: 53-81.

8 Mazzarella G, MacDonald TT, Salvati VM et al: Constitutive activation of the signal transducer and activator of transcription pathway in celiac disease lesions. Am J Pathol 2003; 162: $1845-$ 1855.

9 Monteleone G, Pender SL, Wathen NC, MacDonald TT: Interferon-alpha drives $\mathrm{T}$ cell-mediated immunopathology in the intestine. Eur J Immunol 2001; 31: 2247-2255.

10 Monteleone I, Monteleone G, Del Vecchio Blanco G et al: Regulation of the T helper cell type 1 transcription factor T-bet in coeliac disease mucosa. Gut 2004; 53: 1090-1095.

11 Woolley N, Mustalahti K, Maki M, Partanen J: Cytokine gene polymorphisms and genetic association with coeliac disease in the Finnish population. Scand J Immunol 2005; 61: 51-56.

12 Rioux JD, Karinen $\mathrm{H}$, Kocher $\mathrm{K}$ et al: Genomewide search and association studies in a Finnish celiac disease population: identification of a novel locus and replication of the HLA and CTLA4 loci. Am J Med Genet A 2004; 130: 345-350.

13 Kristiansen OP, Larsen ZM, Pociot F: CTLA-4 in autoimmune diseases - a general susceptibility gene to autoimmunity? Genes Immun 2000; 1: 170-184.

14 van Belzen MJ, Mulder CJ, Zhernakova A, Pearson PL, Houwen $\mathrm{RH}$, Wijmenga C: CTLA4 $+49 \mathrm{~A} / \mathrm{G}$ and CT60 polymorphisms in Dutch coeliac disease patients. Eur J Hum Genet 2004; 12: $782-785$.

15 Zhernakova A, Eerligh P, Barrera P et al: CTLA4 is differentially associated with autoimmune diseases in the Dutch population. Hum Genet 2005; 118: 58-66.

16 Popat S, Hearle N, Hogberg L et al: Variation in the CTLA4/CD28 gene region confers an increased risk of coeliac disease. Ann Hum Genet 2002; 66: 125-137.

17 Revised criteria for diagnosis of coeliac disease: Report of working group of European society of paediatric gastroenterology and nutrition. Arch Dis Child 1990; 65: 909-911.

18 Hunt KA, McGovern DP, Kumar PJ et al: A common CTLA4 haplotype associated with coeliac disease. Eur J Hum Genet 2005; 13: $440-444$

19 Naluai AT, Nilsson S, Samuelsson L et al: The CTLA4/CD28 gene region on chromosome $2 \mathrm{q} 33$ confers susceptibility to celiac disease in a way possibly distinct from that of type 1 diabetes and other chronic inflammatory disorders. Tissue Antigens 2000; 56: $350-355$.

20 Djilali-Saiah I, Schmitz J, Harfouch-Hammoud E, Mougenot JF, Bach JF, Caillat-Zucman S: CTLA-4 gene polymorphism is associated with predisposition to coeliac disease. Gut 1998; 43: 187-189.

21 Clot F, Fulchignoni-Lataud MC, Renoux C et al: Linkage and association study of the CTLA-4 region in coeliac disease for Italian and Tunisian populations. Tissue Antigens 1999; 54: 527-530.

22 Holopainen P, Arvas M, Sistonen P et al: CD28/CTLA4 gene region on chromosome $2 \mathrm{q} 33$ confers genetic susceptibility to celiac disease. A linkage and family-based association study. Tissue Antigens 1999; 53: 470-475.

23 King AL, Moodie SJ, Fraser JS et al: CTLA-4/CD28 gene region is associated with genetic susceptibility to coeliac disease in UK families. J Med Genet 2002; 39: 51-54.

24 King AL, Moodie SJ, Fraser JS et al: Coeliac disease: investigation of proposed causal variants in the CTLA4 gene region. Eur J Immunogenet 2003; 30: 427-432.

25 Mora B, Bonamico M, Indovina $\mathrm{P}$ et al: CTLA-4 +49 A/G dimorphism in Italian patients with celiac disease. Hum Immunol 2003; 64: 297-301.

26 Popat S, Hearle N, Wixey J et al: Analysis of the CTLA4 gene in Swedish coeliac disease patients. Scand J Gastroenterol 2002; 37: $28-31$.

27 Martin-Pagola A, Perez de Nanclares G, Vitoria JC et al: No association of CTLA4 gene with celiac disease in the Basque population. J Pediatr Gastroenterol Nutr 2003; 37: 142-145.

28 Amundsen SS, Naluai AT, Ascher $\mathrm{H}$ et al: Genetic analysis of the CD28/CTLA4/ICOS (CELIAC3) region in coeliac disease. Tissue Antigens 2004; 64: 593-599.

29 Haimila K, Smedberg T, Mustalahti K, Maki M, Partanen J, Holopainen P: Genetic association of coeliac disease susceptibility to polymorphisms in the ICOS gene on chromosome $2 \mathrm{q} 33$. Genes Immun 2004; 5: 85-92.

30 Shuai K, Liu B: Regulation of JAK-STAT signalling in the immune system. Nat Rev Immunol 2003; 3: 900-911.

31 Jacobsen $M$, Schweer D, Ziegler A et al: A point mutation in PTPRC is associated with the development of multiple sclerosis. Nat Genet 2000; 26: 495-499. 